prospective longitudinal designs, systematically ascertained samples and perhaps implicit measures which cover other potentially interesting and clinically relevant cognitive traits such as goal attainment, attributions, self-representations and novelty-seeking.

Power, M. J., Katz, R., McGuffin, P., et al (1994) The Dysfunctional Attitudes Scale (DAS): a comparison of forms $A$ and $B$ and proposal for a new sub-scaled version. Journal of Research in Personality, 28, 263-276.

L. Jones Department of Psychiatry, University of Birmingham, Queen Elizabeth Psychiatric Hospital, Birmingham B29 5JF, UK.

Email: I.a.jones@bham.ac.uk

J. Scott Division of Psychological Medicine, Institute of Psychiatry, London, UK

I. Jones, N. Craddock Department of

Psychological Medicine, Cardiff University, Cardiff, UK doi: 10.I192/bjp.I89.1.85a

\section{What is pathological lying?}

The article by Yang et al (2005) is provocative, thoughtful and intriguing and provided much food for thought. Participants were divided into three groups: liars, normal controls and antisocial controls. Half of those in the liars group were malingerers and the others displayed conning/manipulative behaviour on the Psychopathy Checklist Revised (PCL-R), deceitfulness criteria for DSM-IV antisocial personality disorders or pathological lying as defined in the PCL-R. Yang et al referred to pathological liars specifically in the title of their paper but we are concerned that the definition of liars was so broad and wondered whether the article would not have been better entitled 'Prefrontal white matter in liars'. The authors included individuals with different lying characteristics in a group of pathological liars and this is problematic.

Our recent review (Dike et al, 2005) showed that the term 'pathological lying' has been used differently in the literature from how it was used by Yang et al. Pathological lying is distinct from malingering or the other forms of lying exhibited by those included by Yang $e t$ al in the liars group. We defined pathological lying as 'falsification entirely disproportionate to any discernible end in view, may be extensive and very complicated, and may manifest over a period of years or even a lifetime'. Pathological lying is a repetitive pattern of lying for which an external reason (such as financial gain) often appears absent, and the psychological basis is often unclear. This definition has not been accepted by the psychiatric community but summarises the elements of pathological lying. Interestingly, we found that pathological lying can also be found among successful individuals without a history of criminal behaviour.

We commend Yang et al for investigating the neurobiological basis of lying. Whether the prefrontal white matter changes indicate a causal relationship with lying or just an association is unknown. However, pathological lying per se was not specifically investigated, as suggested.

Dike, C. C., Baranoski, M. \& Griffith, E. E. H. (2005) Pathological lying revisited. Journal of the American Academy of Psychiatry and the Law, 33, 342-349.

Yang, Y., Raine, A., Lencz, T., et al (2005) Prefrontal white matter in pathological liars. British Journal of Psychiatry, 187, 320-325.

C. Dike Department of Psychiatry, Law and Psychiatry Division, Yale University Medical School, New Haven, CT 06519, USA

Email: cd244@email.med.yale.edu

M. Baranoski, E. E. H. Griffith Department of Psychiatry, Law and Psychiatry Division, Yale University Medical School, New Haven,

Connecticut, USA

doi: 10.II92/bjp.I89.1.86

Authors' reply: We wholeheartedly agree with Dike $e t$ al that the definition of 'pathological liar' is vague and confusing. Although pathological lying has been defined in several different ways, no specific psychological test is available. Hence we applied a symptom-based approach and defined individuals as 'liars' if they fulfilled: (a) criteria for pathological lying on the Psychopathy Checklist - Revised (PCL-R), (b) criteria for conning/manipulative behaviour on the PCL-R, (c) the deceitfulness criterion for DSM-IV, or (d) criteria for malingering as reported in a self-report crime interview.

We maintain that our study did investigate at least one form of pathological lying. In a new analysis, we found that $42 \%$ of our liars had psychopathy, antisocial personality disorders or borderline personality disorder. These liars likely correspond to those Healy \& Healy (1926) refer to as 'secondary pathological liars' - people whose lying is a complication of disorders such as those above. The other $58 \%$ of our group, who did not meet this comorbid requirement, probably correspond to the 'primary pathological liars' described by Healy \& Healy people who habitually lie but do not demonstrate symptoms of a clearly defined psychiatric disorder. This new analysis also revealed that liars with or without psychiatric disorders showed significantly increased prefrontal white matter volume compared with antisocial controls $(P=0.003, \quad P=0.01$, two-tailed respectively) and normal controls $(P=0.005$, $P=0.014$ respectively). Although our study is a preliminary attempt to reveal brain abnormalities in people who lie, cheat and deceive we hope that it will stimulate interest in this important but understudied phenomenon.

Healy, W. \& Healy, M. T. (1926) Pathological Lying, Accusation and Swindling. Boston, MA: Little Brown.

Y. Yang Department of Psychology, University of Southern California, Los Angeles, CA 90089, USA. Email: yalingy@usc.edu

A. Raine Department of Psychology, University of Southern California, Los Angeles, California, USA doi: 10.I192/bjp.189.I.86a 